\title{
Analytical Framework for Outage and BER Analysis of the V-BLAST Algorithm
}

\author{
S. Loyka ${ }^{1}$, F. Gagnon ${ }^{2}$
}

\begin{abstract}
Analytical approach to outage and BER analysis of the V-BLAST and D-BLAST algorithms in i.i.d. Rayleigh channel is presented in this paper. Based on the previous results, which allow an exact analytical BER and outage analysis of a $2 \times n$ system in a closed-form, generic case of mxn system is analytically analyzed. While closed-form exact analysis is not feasible, a tight bound for the outage probability is derived and validated using Monte-Carlo simulations. It is shown that the optimal ordering procedure results at a SNR gain of $m$ at the $1^{\text {st }}$ step for $m \leq 4$. We conjecture that this is true for larger $\mathbf{m}$ as well. The results above are also extended to the case of a $D$ BLAST system.
\end{abstract}

\section{INTRODUCTION}

High capacity promise of the MIMO architecture over multipath channels can be achieved using appropriate signal processing algorithm. Few of them have been proposed so far. They differ in complexity and percentage of the full capacity achieved. While the D-BLAST achieves the full MIMO capacity (it was believed for a while that it achieves "significant part" of the full capacity [2], but the recent work of Foschini et al [1] has elegantly demonstrated that, in fact, it achieves the full capacity), its implementation complexity is high. The V-BLAST algorithm, on the contrary, achieves only a part of the full capacity but its implementation capacity is low [3]. Hence, we analyze it in details in this paper. In particular, we develop an analytical framework for the outage and BER analysis of the V-BLAST operating over independent identically-distributed (iid) Rayleigh channel. This framework results in closed-form exact analytical expressions for the outage and BER of the 2xn systems (i.e., with $2 \mathrm{Tx}$ and $\mathrm{n} \mathrm{Rx}$ antennas), which are fully validated using extensive Monte-Carlo simulations. At the moment, we are not able to derive exact closed-form expressions for the generic case of mxn system. However, we present some tight bounds, obtain closed-form expressions based on these bounds and demonstrate, through Monte-Carlo simulations, that the bounds/approximate expressions capture many essential features of the system performance. Finally, based on the results obtained for the V-BLAST, we demonstrate that similar results follow for the D-BLAST.

The major original contribution of the present paper is the extention of the analytical techniques developed for $2 \mathrm{xn} \mathrm{V}$ BLAST systems in [4-6] to the generic case of mxn system ( $\mathrm{m}$ is the number of Tx antennas) and to the D-BLAST system. For completeness, we begin with a brief description of the V-BLAST algorithm and summarize major analytical results for the case of $2 \mathrm{xn}$ systems.

\footnotetext{
${ }^{1}$ School of Information Technology and Engineering (SITE) University of Ottawa, 161 Louis Pasteur, Ottawa, Ontario, Canada K1N6N5 (e-mail: sergey.loyka@ieee.org)

${ }^{2}$ Department of Electrical Engineering, Ecole de Technologie Superieure, 1100, Notre-Dame St. West, Montreal (Quebec), H3C $1 \mathrm{~K} 3$, Canada
}

\section{V-BLAST ALGORITHM ANALYSIS: 2XN SYSTEM}

The V-BLAST algorithm has been discussed in details elsewhere [3]. The main idea of the BLAST architecture is to split the information bit stream into several sub-streams and transmit them in parallel using a set of $\mathrm{Tx}$ antennas (the number of Tx antennas equals the number of sub-streams) at the same time and frequency. At the $\mathrm{Rx}$ side, each $\mathrm{Rx}$ antennas "sees" all the transmitted signals, which are mixed due to the nature of the wireless propagation channel. Using appropriate signal processing at the Rx side, these signals can be unmixed so that the matrix wireless channel is transformed into a set of virtual parallel independent channels (provided that mutlipath is rich enough).

The following basic assumptions are employed in the present paper:

(1) The channel is random, quasistatic (i.e. fixed for every frame of information bits but varying from frame to frame), frequency independent (i.e., negligible delay spread) and with complex AWGN. (2) The Tx signal vector is comprised of individual symbol sub-streams. No space-time coding is employed (however, individual sub-stream coding can be used). (3) The noise vector is comprised of independent AWGN components with equal variance. (4) The Tx signals, noise and channel gains are independent of each other. (5) Perfect channel knowledge is assumed to be available at the receiver. (6) There is no performance degradation due to synchronization and timing errors.

The V-BLAST processing begins with the $1^{\text {st }} \mathrm{Tx}$ symbol and proceeds in sequence to the $\mathrm{m}$-th symbol. When the optimal ordering procedure is employed, the Tx indexing is changed prior to the processing. The main steps of the $\mathrm{V}$ BLAST processing (detection) algorithm are as follows [3]:

1. The interference cancellation step: at the $\mathrm{i}$-th processing step (i.e., when the signal from the i-th transmitter is detected) the interference from the first i-1 transmitters can be subtracted based on the estimations of the Tx symbols (which are actually assumed to be error-free) and the knowledge of the channel matrix $\mathbf{H}$.

2. The interference nulling step: based on the knowledge of the channel matrix, the interference from yet-to-be-detected symbols can be nulled out using the Gramm-Schmidt orthogonalization process (applied to the column vectors of H).

3. The optimal ordering procedure: the order of symbol processing is organized according to their after-processing SNRs in the decreasing order (i.e., the symbol with highest SNR is detected first).

Geometrical framework for the closed-form analysis of the algorithm operation has been discussed in details in [4-6]. Based on those results, the signal fading in the V-BLAST system can be analyzed. In particular, we consider the outage probabilities (i.e., the probability that the after-detection signal power is less than the specified value) and diversity 
order (i.e., the asymptotic slope of the outage probability curve). Ignoring the optimal ordering procedure, it can be proved that the diversity order at the $i$-th step is $(n-m+i)$. Hence, the maximum ratio combining (MRC) outage probability of appropriate order can be used. Consequently, all the results for BER of MRC hold true in the case of VBLAST as well.

However, the optimal ordering procedure "mixes" things up and makes the analysis more challenging. Fortunately, the approach above can be extended to account for the optimal ordering $[5,6]$. We note that in the case of $2 x n$ system the optimal ordering (after the interference nulling) reduces to the following:

$$
s_{1}=(\sin \varphi)^{2} \max \left[\left|\mathbf{h}_{1}\right|^{2},\left|\mathbf{h}_{2}\right|^{2}\right]
$$

where $s_{1}$ is the signal power after the optimal ordering, $\mathbf{h}_{1 \perp}$ $\left(\mathbf{h}_{2 \perp}\right)$ is the part of $\mathbf{h}_{1}\left(\mathbf{h}_{2}\right)$ orthogonal to $\mathbf{h}_{2}\left(\mathbf{h}_{1}\right)$, and $\varphi$ is the angle between $\mathbf{h}_{1}$ and $\mathbf{h}_{2}$. In fact, (1) tells us that the optimal ordering for $2 \mathrm{xn}$ system is to detect first the substream with the largest before-detection power. While $\left|\mathbf{h}_{1 \perp}\right|^{2}$ and $\left|\mathbf{h}_{2 \perp}\right|^{2}$ are $\chi_{2 n-2}^{2}$ (i.e., (n-1)-th diversity order), taking the maximum does not result in doubling the diversity order because they are not independent, as $\sin \varphi$ at the right-hand side of (1) indicates.

Using the fact that the distribution of $\max \left[\left|\mathbf{h}_{1}\right|^{2},\left|\mathbf{h}_{2}\right|^{2}\right]$ is $F_{n}^{2}(x)$, where $F_{n}(x)=\operatorname{Pr}\left[\left|\mathbf{h}_{1}\right|^{2}<x\right]=1-e^{-x} \sum_{k=0}^{n-1} \frac{x^{k}}{k !}$ is n-th order MRC distribution, the distribution $P_{1}(x)=\operatorname{Pr}\left[s_{1}<x\right]$ of $\mathrm{S}_{1}$ can be presented in the following form:

$$
P_{1}(x)=\int_{0}^{\pi / 2} F_{n}^{2}\left(\frac{x}{\sin ^{2} \varphi}\right) f_{\varphi}(\varphi) d \varphi
$$

where $f_{\varphi}(\varphi)$ is the p.d.f. of $\varphi$, which can be shown to be

$$
f_{\varphi}(\varphi)=2(n-1)(\sin \varphi)^{2 n-3} \cos \varphi
$$

This p.d.f. is illustrated in Fig. 1 for $n=2,3$, 4. The most probable direction shifts to the right $\left(90^{\circ}\right)$ when $n$ increases, starting from $45^{\circ}$ for $n=2$. This is what should be intuitively expected because $\mathbf{h}_{1 \|} \sim \chi_{2}^{2}$ and $\left|\mathbf{h}_{1 \perp}\right|^{2} \sim \chi_{2 n-2}^{2}$, and, hence, the most probable value of the ordinate $\left(\left|\mathbf{h}_{1 \perp}\right|\right)$ is greater than the most probable value of the abscissa $\left(\left|\mathbf{h}_{1 \|}\right|\right)$ (for $n>2$ ), and the former increases with $n$ while the later is fixed.

Using (2) and (3), the outage probability $P_{1}(x)$ can be expressed in a closed form as $P_{1}(x)=1-p_{1}(x) e^{-x}+p_{2}(x) e^{-2 x}$, where $p_{1}(x)$ and $p_{2}(x)$ are polynomials of degree at most (n-2) and (2n-3) correspondingly (see $[5,6]$ for the details). The asymptotic behavior of the outage probability can be shown to be

$$
P_{1}(x) \approx(x / 2)^{n-1} /(n-1) !, \quad x \rightarrow 0
$$

Comparing it with (n-1)-order MRC asymptotic behavior, $F_{M R C}(x) \approx x^{n-1} /(n-1)$ !, we conclude that the effect of the optimal ordering at the $1^{\text {st }}$ detection step is to increase SNR

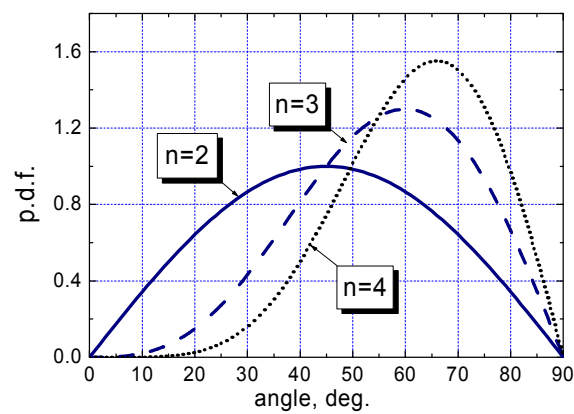

Figure 1. p.d.f. of $\varphi$ for various numbers of receive antennas (n).

by $3 \mathrm{~dB}$ rather than to increase the diversity order. This is the consequence of correlation between $\mathbf{h}_{1 \perp}$ and $\mathbf{h}_{2 \perp}$.

The conditional outage probability at the $2^{\text {nd }}$ detection step (conditioned on no detection error at the $1^{\text {st }}$ step) is given by

$$
P_{2}(x)=\operatorname{Pr}\left[s_{2}<x\right]=F_{n}(x)\left[2-F_{n}(x)\right]
$$

The effect of optimal ordering at the $2^{\text {nd }}$ detection step is to increase the outage probability twice. This is the "price" to pay for the increased SNR at the $1^{\text {st }}$ step. Note that these are exact expressions for the outage probabilities.

Using the outage probabilities above, average BER can be found at each detection step for various modulation formats in a straightforward way (note that only conditional outage probability at the $2^{\text {nd }}$ step is required to find the total average BER). Detailed results, including exact closed-form expressions for the average BER, are presented in $[5,6]$ and omitted here due to the lack of space. It can be shown that for moderate to high SNR, $1^{\text {st }}$ step BER is dominant and the effect of error propagation is negligible (i.e., second-order one).

\section{V-BLAST ALGORITHM ANALYSIS: MXN SYSTEM}

We now generalized the previous results to the case of mxn system. The generalization is highly non-trivial and presents serious difficulties, which we resolve at the moment using various bounds and approximations.

$1^{\text {st }}$ step outage probability can be found in this case using a generalized from of (2) and (3):

$$
\begin{aligned}
& P_{1}(x)=\int_{0}^{\pi / 2} \ldots \int f_{\varphi}\left(\varphi_{1}, \ldots \varphi_{m}\right) \prod_{i=1}^{m} F_{n}\left(\frac{x}{\sin ^{2} \varphi_{i}}\right) d \varphi_{i} \\
& f_{\varphi}(\varphi)=2(m-1) C_{n-1}^{m-1} \sin ^{2(n-m)+1} \varphi \cdot \cos ^{2 m-3} \varphi
\end{aligned}
$$

where $f_{\varphi}\left(\varphi_{1}, \ldots \varphi_{m}\right)$ is the joint pdf of $\left\{\varphi_{1}, \ldots \varphi_{m}\right\}, \varphi_{i}$ being the angle between $\mathbf{h}_{i}$ and the sub-space spanned all the other column vectors, $f_{\varphi}(\varphi)$ is the marginal pdf, and $C_{n}^{m}$ is the binomial coefficient. Note that $f_{\varphi}\left(\varphi_{1}, \ldots \varphi_{m}\right)$ is symmetric with respect to $\left\{\varphi_{1}, \ldots \varphi_{m}\right\}$ (i.e., any two angles can be exchanged without affecting the pdf). The angles are neither independent nor fully correlated, which makes it very difficult to find the joint pdf required in (6). To this end, we use the Holder inequality and derive the following bounds:

$$
P_{1}(x) \leq \int_{0}^{\pi / 2} f_{\varphi}(\varphi) F_{n}^{m}\left(\frac{x}{\sin ^{2} \varphi}\right) d \varphi \leq \int_{0}^{\pi / 2} f_{\varphi}(\varphi) F_{n}\left(\frac{x}{\sin ^{2} \varphi}\right) d \varphi
$$


While the $2^{\text {nd }}$ bound is loose, the $1^{\text {st }}$ one is, as we show later on, quite tight. Additionally, since $\left\{\varphi_{1}, \ldots \varphi_{m}\right\}$ are exchangeable random variables which are known to have non-negative correlation, it can be shown that

$$
\left(\int_{0}^{\pi / 2} f_{\varphi}(\varphi) F_{n}\left(\frac{x}{\sin ^{2} \varphi}\right) d \varphi\right)^{m} \leq P_{1}(x)
$$

After some manipulations, the $1^{\text {st }}$ bound in (8) can be presented as:

$$
B_{1}(x)=\beta\left\{\sum_{l=0}^{m} \alpha_{l}\left(J_{3 l}+J_{4 l}\right)+\sum_{l=2}^{m} \alpha_{l} J_{2 l}\right\}
$$

where $\beta=(-1)^{m-2}(m-1) C_{n-1}^{m-1}, \alpha_{l}=(-1)^{l} C_{m}^{l}$,

$$
J_{2 l}=e^{-l x}(l x)^{n-m+1} \sum_{p=0}^{l(n-1)-n+m-2} a_{p l}(l x)^{p}
$$

$J_{3 l}=(-1)^{n+1} e^{-l x}(-l x)^{n-m+1} \sum_{p=0}^{m-3} b_{p}(-l x)^{p}, J_{4 l}=e^{-l x} \sum_{p=0}^{n-2} d_{p}(-l x)^{p}$

$a_{p l}=\sum_{k=\max [0, m-2-p]}^{m-2} \frac{(-1)^{k} C_{m-2}^{k}}{(p+k-m+2) !} \sum_{i=\max [0, p-m+2]}^{l(n-1)-n} c_{i+n, l} l^{-i-n}(k+i) !$

$b_{p}=\sum_{k=0}^{p} \frac{(-1)^{k} C_{m-2}^{k-p+m-2}}{k !} \sum_{i=0}^{m-p-3} \frac{(k+i) !}{(i+p+n-m+2) !}$

$d_{p}=\sum_{k=0}^{\min [m-2, n-2-p]}(-1)^{k}(n-k-p-2) ! C_{m-2}^{k} \sum_{i=0}^{p} \frac{(-1)^{i}}{i !(n-k-i-1) !}$

$c_{i, l}=\sum_{i_{1}+\ldots+i_{l}=i} \frac{1}{i_{1} ! \ldots i_{l} !}, 0 \leq i_{1}, \ldots i_{l} \leq n-1$

While the expression for $a_{p l}, b_{p}, d_{p}$ may appear complicated, they can be evaluated in advanced (i.e., a table of coefficients is built for a given order of the system) and do not need to be changed during simulations. Note that the bound is presented as a product of exponents and polynomials of finite order (which depends on the system order) and, hence, the procedure is very efficient numerically. Note also that (10) reduces to the known case of $m=2$, as it should be.

To get some insight and to evaluate the bound accuracy, we further consider $3 \times 3$ system.

\section{A. Outage of $3 \times 3 \mathrm{~V}$-BLAST}

The $1^{\text {st }}$ step outage is bounded as

$$
\begin{aligned}
P_{1}(x) & \leq B_{1}(x)=1-3 e^{-x}+e^{-2 x}\left(3+15 x / 8+3 x^{2} / 8\right) \\
& -e^{-3 x}\left(1+110 x / 81+7 x^{2} / 9+2 x^{3} / 9+x^{4} / 36\right)
\end{aligned}
$$

The asymptotic behavior of the bound is

$$
B_{1}(x) \approx 335 x / 648 \approx x / 2, x \rightarrow 0
$$

which is the same as the asymptotic outage probability of the $2 \times 2$ system [5,6]. $1^{\text {st }}$ order diversity and $3 \mathrm{~dB}$ gain due to optimal ordering are apparent (this $3 \mathrm{~dB}$ gain transforms asymptotically into $3 \mathrm{~dB}$ gain in terms of the average BER).

The $2^{\text {nd }}$ step outage cannot be easily evaluated since the ordering procedure at the $1^{\text {st }}$ step affects the channel statistics at the $2^{\text {nd }}$ step. We evaluate the conditional outage probability at the $2^{\text {nd }}$ step (i.e., conditioned on no detection error at the $1^{\text {st }}$ step - this is what we need to evaluate the total outage probability and BER [6]). As an approximation, we assume that the channel statistics at the $2^{\text {nd }}$ step is not affected by the optimal ordering at the $1^{\text {st }}$ step (i.e., the channel coefficients are still i.i.d. complex Gaussian). Under this assumption, the $2^{\text {nd }}$ step outage probability is the same as that of a $2 \times 3$ system at the $1^{\text {st }}$ step (since the first bit stream has been detected and eliminated at the $1^{\text {st }}$ step), whose outage is $[5,6]$,

$$
P_{2}(x)=1-2 e^{-x}(1+x)+e^{-2 x}\left(1+2 x+9 x^{2} / 8+x^{3} / 4\right)
$$

Its asymptotic behavior is

$$
P_{2}(x) \approx x^{2} / 8, x \rightarrow 0
$$

Second-order diversity is obvious.

The $3^{\text {rd }}$ step conditional outage probability can be evaluated in a similar way. Assuming no change in the channel statistics due to the ordering in the first two steps, it is the same as that of a $2 \times 3$ system at the second step,

$$
P_{3}(x)=F_{3}(x)\left[2-F_{3}(x)\right]
$$

Its asymptotic behavior is $P_{3}(x) \approx 2 F_{3}(x) \approx x^{3} / 3$, which indicates the $3^{\text {rd }}$ order diversity.

Extensive Monte-Carlo simulations have been carried out ! to evaluate the accuracy of the bound and approximations involved. The results are shown in Fig. 2. Clearly, the $1^{\text {st }}$ step bound is quite accurate (given its simple nature) and it underestimates the performance by $2 \mathrm{~dB}$. The actual asymptotic behavior of the outage probability is

$$
P_{1}(x) \approx x / 3, x \rightarrow 0
$$

We conjecture that in general, the asymptotic outage probability is

$$
P_{1}(x) \approx x / m, x \rightarrow 0
$$

Note that it is true for $2 \mathrm{xn}$ system $[5,6]$, our simulations here confirm it for $3 \times 3,3 \times 4$ and $4 \times 4$ systems.

The $2^{\text {nd }}$ step performance is overestimated by $3 \mathrm{~dB}$. However, as fig. 2 demonstrates, it is predicted extremely well by the $2^{\text {nd }}$ order MRC outage curve. We attribute this to the joint effect of two opposite factors: 1) performance loss at the $2^{\text {nd }}$ step due to optimal ordering at the $1^{\text {st }}$ (the same as for $2 \mathrm{xn}$ system), and 2) performance improvement due to the $2^{\text {nd }}$ step optimal ordering. Apparently, this two effects compensate each other and the resulting outage is the same as that of $2^{\text {nd }}$ order MRC.

The $3^{\text {rd }}$ step performance is estimated quite accurately by the approximate expression (15) (within $1 \mathrm{~dB}$ ). MRC outage curve would provide worse approximation in that case.

\section{B. Outage of $4 \times 4 \mathrm{~V}$-BLAST}

The validity of the approximations above is not limited to a $3 \times 3$ system. As an example, we use the same approximations to analyze $4 \times 4$ system. Fig. 3 shows the outage probability at first 3 steps. The $1^{\text {st }}$ step bound and its asymptotic behavior are obtained using (10). The asymptotic behavior of the $1^{\text {st }}$ step outage is given by (17) for $m=4$.

The $2^{\text {nd }}$ step outage has been analytically estimated using the $1^{\text {st }}$ step outage of a $3 \times 4$ system, which is within $1.5 \mathrm{~dB}$ of the actual performance. Note that it is not the same as MRC anymore. However, the $3^{\text {rd }}$ order performance is virtually the same as that of $3^{\text {rd }}$ order MRC. The analytic estimation of the performance (using $1^{\text {st }}$ step outage of a $2 \times 4$ system) overestimates it by approximately $3 \mathrm{~dB}$. We attribute this to the effect of the optimal ordering at the $1^{\text {st }}$ and $2^{\text {nd }}$ steps. 
It should be noted that using the analytical approximations for the outage probabilities, the average BER can be evaluated in a straightforward way. Closed-form BER expressions can also be derived for various modulation formats (in the same way as in $[5,6]$ ).

\section{Outage Probabilities AND BER of the D-BLAST ALGORITHM}

The D-BLAST algorithm is an attractive MIMO solution (despite of its complexity) since it achieves the full MIMO capacity. Outage probabilities at various steps of the DBLAST algorithm can be obtained using the results above. In particular, we observe that all the $\mathrm{Tx}$ antennas are periodically "rotated" with respect to the transmitters with which they are connected [2]. Hence, in terms of the present performance analysis, it means that the signals sent by, say, $1^{\text {st }} \mathrm{Tx}$ are detected at various steps of the detection process (form step 1 to the last one), and not at some particular step determined by the optimal ordering procedure, as it was the case for the V-BLAST, when the connection of a Tx to an antenna was fixed and channel-independent. Hence, the average outage probability (averaged out for many symbols) of the D-BLAST is the average of step outage probabilities and it is the same for all the Txs (due to the problem symmetry):

$$
P(x)=\frac{1}{m} \sum_{i=1}^{m} P_{i}
$$

where $P_{i}$ are the i-th step outage of the V-BLAST. Using the exact closed-from expressions given above, the average outage probability of the D-BLAST can be immediately obtained in a closed-form (ignoring the effect of error propagation). It should be noted that, using the Bayes formula, the error propagation can be accounted for as well and the total outage probability can be derived using the conditional outage probabilities above. Using the analytical approximations above, similar closed-form expressions can be derived for mxn D-BLAST. Furthermore, using these outage probabilities, closed-from expressions can be derived for the average BER of the D-BLAST. Note that this analysis also gives a time-domain structure of the outage probabilities (i.e., its variation from symbol to symbol).

It is instructive to consider the asymptotic behavior of (18), i.e. when the average SNR is high and the $1^{\text {st }}$ step outage probability is dominant (recall that the diversity order increases with the step number),

$$
P_{1} \gg P_{2} \ldots \gg P_{m}
$$

In this case,

$$
P \approx P_{1} / m
$$

Clearly, the effect of antenna "rotation" is to decrease asymptotically the $1^{\text {st }}$ step outage $m$ times. Recall that for the V-BLAST system in a high-SNR mode the total outage probability is dominated by the $1^{\text {st }}$ step one, $P_{V-B L A S T} \approx P_{1}$ [6]. Clearly, D-BLAST outperforms V-BLAST $m$ times. Note, however, that the $2^{\text {nd }}$ and higher step outage probabilities will be increased. This, however, does not present a significant problem because the total outage probability is dominated by the $1^{\text {st }}$ step one (asymptotically) and, hence, it is crucial to improve the latter.

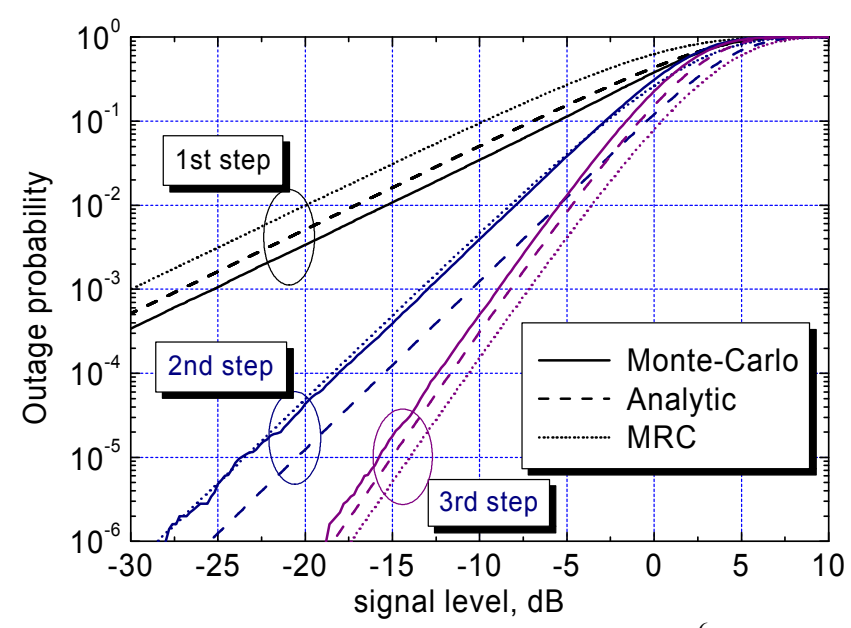

Figure 2. Outage probabilities of $3 \times 3$ V-BLAST. $5 * 10^{6}$ trials have been used for Monte-Carlo simulations.

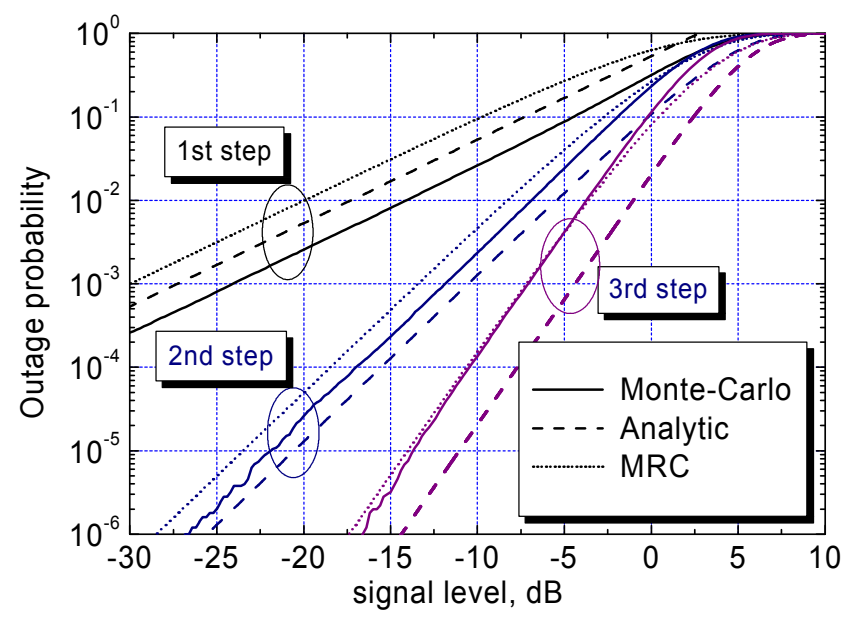

Figure 3. Outage probabilities of $4 \times 4$ V-BLAST. $5 * 10^{6}$ trials have been used for Monte-Carlo simulations.

\section{REFERENCES}

[1] G.J. Foschini et al, Analysis and Performance of Some Basic Space-Time Architectures, IEEE Journal Selected Areas Comm., v. 21, N. 3, pp. 281-320, April 2003.

[2] G.J Foschini, 'Layered space-time architecture for wireless communication in a fading environment when using multiple antennas', Bell Lab. Tech. J., vol. 1, N. 2, pp. 41-59, 1996.

[3] G.J Foschini et al, Simplified Processing for High Spectral Efficiency Wireless Communication Employing Multi-Element Arrays, IEEE Journal on Selected Areas in Communications, v. 17, N. 11, pp. 1841-1852, Nov. 1999.

[4] S. Loyka, F. Gagnon, Performance Analysis of the VBLAST Algorithm: An Analytical Approach, 2002 International Zurich Seminar on Broadband Communications, Feb. 19-21, Zurich, Switzerland, pp. 5_1-5_6.

[5] S. Loyka, V-BLAST Outage Probability: Analytical Analysis, IEEE Vehicular Technology Conference, 24-28 September 2002, Vancouver, Canada.

[6] S. Loyka, F. Gagnon, Performance Analysis of the VBLAST Algorithm: an Analytical Approach, IEEE Trans. Wireless Comm., accepted, 2003.

[7] F.R. Gantmaher, Theory of Matrices, Nauka, Moscow, 1988 (in Russian).

[8] G.A. Korn, T.M. Korn, Mathematical Handbook for Scientists and Engineers, Dover, New York, 2000.

[9] M. Abramowitz, I. Stegun, Handbook of Mathematical Functions, National Bureau of Standards, 1964. 